arthritis ( $81.7 \%$ male vs $73.8 \%$ female). There was a higher prevalence of fibromyalgia among females (20\% vs $7.0 \%$ male). Overall, females reported worse self-perceived health $(67.0 \%$ vs $51.4 \%, \mathrm{p}<0.001)$, higher risk of anxiety $(59.5 \%$ vs $48.1 \%, p<0.001)$, and depression ( $48.0 \%$ vs $37.2 \%, p<0.001)$. Females reported a greater increase in smoking $(26.5 \%$ vs $17.5 \%, p=0.001)$, although they were less likely to drink alcohol ( $34.5 \%$ vs $25.4 \%, p=0.013)$, and also engaged less in physical activity $(53.0 \%$ vs $60.3 \%, p=0.045)$. Overall, females were more likely to keep their scheduled rheumatology appointment $(43.3 \%$ vs $34.1 \%$ of males $(p=0.049$; Table 1) with a higher proportion of females having their rheumatic treatment changed $(17.0 \%$ vs $10.7 \%, p=0.005)$.

Conclusion: The first wave of the COVID-19 pandemic and the containment measures have worsened self-perceived health status of patients with RMDs, affecting genders differently. Females reported worse psychological health and life habits such as increased smoking and reduced physical activity, while males increased their alcohol consumption and were less likely to attend their rheumatology appointments.

Acknowledgements: This study was supported by Novartis Pharma AG. We would like to thank all patients that completed the survey as well as all of the patient organisations that participated in the REUMAVID study including: the Cyprus League Against Rheumatism (CYPLAR) from Cyprus, the Association Française de Lutte Anti-Rhumatismale (AFLAR) from France, the Hellenic League Against Rheumatism (ELEANA) from Greece, the Associazione Nazionale Persone con Malattie Reumatologiche e Rare (APMARR) from Italy, the Portuguese League Against Rheumatic Diseases (LPCDR), from Portugal, the Spanish Federation of Spondyloarthritis Associations (CEADE), the Spanish Patients' Forum (FEP), UNiMiD, Spanish Rheumatology League (LIRE), Andalusian Rheumatology League (LIRA), Catalonia Rheumatology League and Galician Rheumatology League from Spain, and the National Axial Spondyloarthritis Society (NASS), National Rheumatoid Arthritis (NRAS) and Arthritis Action from the United Kingdom.

Disclosure of Interests: Marco Garrido-Cumbrera: None declared, Helena Marzo-Ortega Speakers bureau: AbbVie, Biogen, Celgene, Janssen, Lilly, Novartis, Pfizer, Takeda and UCB, Consultant of: AbbVie, Celgene, Janssen, Lilly, Novartis, Pfizer and UCB, Grant/research support from: Janssen and Novartis, Laura Christen Employee of: Novartis Pharma AG, Loreto Carmona: None declared, José Correa-Fernández: None declared, Sergio Sanz-Gómez: None declared, Pedro Plazuelo-Ramos: None declared, Souzi Makri Grant/research support from: Novartis, GSK and Bayer, Elsa Mateus Grant/research support from: Pfizer, grants from Lilly Portugal, Sanofi, AbbVie, Novartis, Grünenthal S.A., MSD, Celgene, Medac, Janssen-Cilag, Pharmakern, GAfPA., Serena Mingolla: None declared, KATY ANTONOPOULOU: None declared, LAURENT GRANGE: None declared, Clare Jacklin Grant/research support from: Abbvie, Amgen, Biogen, Eli Lilly, Gilead, Janssen, Pfizer, Roche, Sanofi \& UCB., Dale Webb Grant/research support from: AbbVie, Biogen, Janssen, Lilly, Novartis and UCB., Shantel Irwin: None declared, Victoria Navarro-Compán Grant/research support from: Abbvie, BMS, Janssen, Lilly, MSD, Novartis, Pfizer, Roche, and UCB DOI: 10.1136/annrheumdis-2021-eular.2417

\section{AB0678 RATES OF SURGICAL PROCEDURES OF THE KNEE AND HIP DURING THE "FIRST WAVE" OF COVID 19 IN SWEDEN}

A. Dell'isola ${ }^{1}$, A. Kiadaliri ${ }^{1}$, A. Turkiewicz ${ }^{1}$, V. Hughes ${ }^{1}$, K. Magnusson ${ }^{1,2}$ J. Runhaar ${ }^{3}$, S. M. A. Bierma-Zeinstra ${ }^{3}$, M. Englund ${ }^{1} .{ }^{1}$ Lund University, Clinical Epidemiology Unit, Orthopedics, Department of Clinical Sciences Lund, Lund, Sweden; ${ }^{2}$ Norwegian Institute of Public Health, Cluster for Health Services Research, Oslo, Norway; ${ }^{3}$ Erasmus MC, University Medical Center, Department of General Practice, Rotterdam, Netherlands

Background: Many countries imposed lockdowns in March 2020, in anticipation of the "first wave" of COVID-19 and the massive healthcare resources required to meet its acute medical needs. Sweden adopted a different strategy to contain the epidemic, opting for non-binding recommendations. Nonetheless, elective and acute surgical procedures in health care may have been affected.

Objectives: To investigate the effect of the "first-wave" of COVID-19 and the government's response in Sweden on the rates of total joint replacements (TJR), arthroscopies, and fracture surgeries of the knee and hip.

Methods: We used register data for the entire population of Skåne, the southernmost region in Sweden with 1.3 million inhabitants (13\% of the total Swedish population). We identified all residents aged $\geq 18$ years who between $1^{\text {st }}$ January 2015 and $31^{\text {st }}$ November 2020 underwent any of the following surgical procedures of the knee or hip: TJR (TJR due to fracture excluded), arthroscopy, and surgery due to fracture (including TJR). To demarcate pre-event and post-event periods, we established a differentiation point corresponding to mid-March 2020, the timepoint at which the the Swedish Public Health Agency began recommending social distancing, working from home, distance learning for secondary schools and universities, At the aggregate level, we modelled the number of surgeries per 10,000 adults from January 2015 up to September 2020. We did an interrupted time-series (ITSA) analysis using segmented ordinary least-squares regression to estimate changes in the levels and trends of surgical procedures compared to pre-COVID-19 levels, adjusting for seasonal variations. The month of March was treated as a "phase-in" period to give time for the new recommendations to be implemented. In addition we estimated the absolute and relative difference (with its $95 \%$ confidence interval $[\mathrm{Cl}]$ ) between the predicted and the counterfactual scenario in the monthly number of surgeries from April 2020, where the counterfactual is the rate of surgery that would have been expected if COVID-19 had not happened. To account for the possibility that other co-occurring events may be responsible for the observed changes, we assessed changes in the number of surgeries due to fractures, which are normally treated as emergencies that cannot be cancelled or rescheduled, and thus should be less affected, at least by policies at the hospital level.

Results: We identified a total of 20,831 TJRs, 12,156 arthroscopies and 15,041 fracture surgeries of the knee or hip over the study period. The monthly rate of surgeries and ITSAs are presented in Figure 1, with the pre-COVID period starting from February 2019 for readability (Figure 1). The results suggest that in April 2020 there was a decrease of $2.08(95 \% \mathrm{Cl} 1.81 ; 2.35)$ TJRs per 10,000 adults which corresponds to a decrease of $74 \%(95 \% \mathrm{Cl} 65 \%$; $85 \%)$ when compared to the counterfactual scenario. This was followed by a positive trend signifying a monthly increase of $0.36(95 \% \mathrm{Cl} 0.31 ; 0.40)$ TJRs per 10,000 adults. The rate of arthroscopies followed a similar pattern with a decrease of $0.55(95 \% \mathrm{Cl} 0.39 ; 0.71)$ arthroscopies per 10,000 adults in April, which corresponds to a $49 \%$ decrease $(95 \% \mathrm{Cl} 28 \%$; $63 \%)$ followed by a positive trend signifying a monthly increase of $0.11(95 \% \mathrm{Cl}$ $0.07 ; 0.15$ ) arthroscopies per 10,000 adults. The rate of surgery due to knee or hip fractures showed no decrease in April and was followed by a negative trend signifying a monthly decrease of $0.03(95 \% \mathrm{Cl} 0.002 ; 0.04)$ surgeries per 10,000 adults Conclusion: In Sweden, we observed a marked decrease in the number of typical elective knee and hip surgeries such as TJRs and arthroscopies, following the gov ernment's response to Covid-19. We then observed a slow but steady recovery that brought the rates of procedures towards expected levels by Fall 2020, before the "second wave" hit the country. The number of acute fracture surgeries showed no sharp drop, instead showing a steady and slow decline potentially due to reduction in commuting and in physical activities linked to recommendations of social

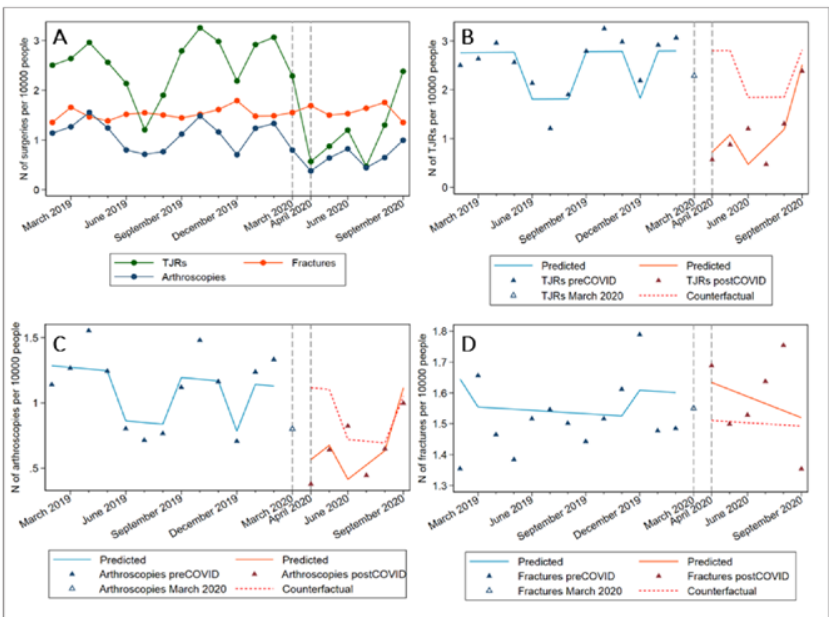

Figure 1: Monthly incidence of surgeries per 10,000 Skåne inhabitants aged $>18$

$A$ : Monthly incidence for TJR, arthroscopies and fracture-related surgeries. Interrupted time series for TJR, C: arthroscopies, and D: fracture-related surgeries. Pre-COVID data is presented from February 2019 onwards for readability.

Disclosure of Interests: None declared

DOI: 10.1136/annrheumdis-2021-eular.2494

\section{AB0679 \\ NAILFOLD VIDEOCAPILLAROSCOPY RESULTS IN COVID-19 PATIENTS RECOVERED FROM DIFFERENT DISEASE SEVERITY}

E. Gotelli ${ }^{1}$, P. F. Bica ${ }^{1}$, T. Aloe', ${ }^{2}$ A. Sulli ${ }^{1}$, M. Grosso ${ }^{2}$, C. Pizzorni ${ }^{1}$, F. Cattelan ${ }^{1}$, S. Paolino ${ }^{1}$, E. Barisione ${ }^{2}$, V. Smith ${ }^{3}$, M. Cutolo ${ }^{1} .{ }^{1}$ Laboratory of Experimental Rheumatology and Academic Division of Clinical Rheumatology, Department of Internal Medicine and Specialties, University of Genova, IRCCS San Martino Polyclinic, Genova, Italy; ${ }^{2}$ Interventional Pneumology Unit, IRCCS San Martino Polyclinic, Genoa, Italy; ${ }^{3}$ Department of Rheumatology - Department of Internal Medicine - Unit for Molecular Immunology and Inflammation, VIB Inflammation Research Center (IRC), Ghent University Hospital, Ghent, Belgium

Background: COVID-19 is a multifaceted condition with a wide range of clinical manifestations, including microvascular/endothelial dysfunction, that starts in the early phase of the disease and may become dramatically harmful in the late stage, causing a massive pro-thrombotic state. Nailfold videocapillaroscopy (NVC) is the most used tool to identify microvascular status in a large spectrum 
of diseases [1]. Recently, non-specific NVC abnormalities have been described in a cohort of COVID-19 patients (no controls used) [2]

Objectives: To assess microvascular damage in recovered COVID-19 patients (range of 40-270 days from recovery) by considering the previous severity of the disease, and, as mandatory, the comparison with matched individuals suffering from primary Raynaud's phenomenon (PRP) and healthy volunteers (HV).

Methods: NVC investigations were performed during standard clinical assessments in forty-four recovered COVID-19 patients (mean age $58 \pm 14$ years, mean days from disease onset $129 \pm 54$, mean days from disease recovery $106 \pm 52$ ), twenty-two patients with PRP (mean age $60 \pm 15$ years, mean years from disease onset $11 \pm 10$ ) and twenty-two HV (mean age 60 \pm 14 years). COVID-19 patients were divided into two subgroups, according to the need of oxygen supplementation: twenty-two patients with severe lung involvement (need of Continuous Positive Airways Pressure and/or mechanical ventilation, mean age $57 \pm 12$ years) vs twenty-two patients with mild-moderate lung involvement (need of Venturi mask or no need of oxygen supplementation, mean age $59 \pm 15$ years). Clinical and demographic data of all the enrolled subjects were collected, during NVC examination. The following capillaroscopic parameters were evaluated: capillary number, dilated capillaries, giant capillaries, microhemorrhages, angiogenesis, disorganization of the microvascular array. A validated semiquantitative scoring (0-3) was adopted for NVC abnormalities [3-5]. Statistical analysis was carried out by non-parametric tests.

Results: After COVID-19 recovery, no statistically significant difference was observed between COVID-19 patients and control groups of subjects concerning the score for the following NVC parameters: dilated capillaries, giant capillaries, disorganization of the microvascular array, angiogenesis. However, the capillary number per linear millimeter was significantly lower in COVID-19 patients $(8.3 \pm 0.9)$ than in PRP $(8.8 \pm 0.7, p=0.05)$ and HV $(9.3 \pm 0.6, p<0.0001)$. Surprisingly, recovered COVID-19 patients showed significantly less microhemorrhages (score $0.4 \pm 0.3$ ) than subjects of the other groups (PRP $0.6 \pm 0.5, p=0.01$; HV $0.6 \pm 0.6, p=0.05)$. In particular, recovered patients who had more severe COVID19 showed less microhemorrhages than patients with mild/moderate disease (score $0.18 \pm 0.4$ vs $0.36 \pm 0.5$ ), but this didn't reach the statistical significance $(p=0.18)$. On the other hand, patients recovered from severe SARS-CoV-2 infection also showed higher rate of angiogenesis $(0.18 \pm 0.4)$ than patients with mild/ moderate disease (no case, $\mathrm{p}=0.04$ ).

Conclusion: COVID-19 doesn't seem to significantly induce, in short-term, specific alterations in peripheral microvascular array as evaluated by NVC, despite the severity of the disease, except for a significant reduction of the absolute number of nailfold capillaries. The topic needs longer time of evaluation and larger number of COVID-19 recovered cases to also assess the role of concomitant therapies. REFERENCES:

[1] Ingegnoli F et al. Curr Rheumatol Rev. 2018;14:5-11.

[2] Natalello G et al. Microvasc Res. 2021;133:104071.

[3] Smith V et al. Autoimmun Rev 2020;19:102458.

[4] Cutolo M et al. Clin Rheumatol. 2019;38:2293-2297.

[5] Sulli A et al. Ann Rheum Dis. 2008;67:885-7.

Disclosure of Interests: None declared

DOI: 10.1136/annrheumdis-2021-eular.2526

\section{AB0680 SARCOPENIA RATE IN COVID-19 SURVIVORS}

D. Levy ${ }^{1}$, M. Giannini ${ }^{1,2}$, W. Oulehri ${ }^{3}$, M. Riou ${ }^{1}$, C. Marcot ${ }^{4}$, A. Meyer ${ }^{1,2,5}$, B. Geny ${ }^{1,2}$. 'Hôpitaux Universitaires de Strasbourg, Physiology, Strasbourg, France; ${ }^{2}$ Université de Strasbourg, Fédération de Médecine Translationnelle de Strasbourg (FMTS), Faculté de Médecine, EA 3072 «Mitochondrie, Stress oxydant et Protection Musculaire », Strasbourg, France; ${ }^{3} \mathrm{Hôpitaux}$ Universitaires de Strasbourg, Service de Réanimation Chirurgicale, Strasbourg, France; ${ }^{4}$ Hôpitaux Universitaires de Strasbourg, Service de Pneumologie, Strasbourg, France; ${ }^{5}$ Hôpitaux Universitaires de Strasbourg, Service de Rhumatologie, Strasbourg, France

Background: COVID-19 survivors may have high risks of developing sarcopenia, which is associated to handicap, poor quality of life, higher healthcare costs, increased risk of falls, increased mortality (1).

Objectives: To assess sarcopenia rate (which has not been described yet) following COVID-19 hospitalization and to highlights features associated with sarcopenic vs. non-sarcopenic patients.

Methods: Major confirmed COVID-19 patients undergoing intensive care unit (ICU) or Pneumology Department admission in Strasbourg University Hospital between March and June 2020 were prospectively included. Muscle and cardio-respiratory evaluations were performed 3 months after discharge. Sarcopenic patients were compared to non-sarcopenic ones. A second muscle assessment 6 months after discharge was performed in patients with pathologic muscle tests. Results: 127 patients were included, 39 (30.7\%) not requiring ICU care, 88 (69.3\%) requiring ICU care. The cohort consisted of $71 \%$ male patients, with a median age of 63 years [28-82]. Forty-one-percent were obese $\left(\mathrm{BMl}>30 \mathrm{~kg} / \mathrm{m}^{2}\right)$. Most individuals had preexisting comorbidities (82 patients, $70 \%$ ), mainly hypertension and diabetes mellitus. The median total hospital stay duration was 22 days and up to 89 days. At the 3 months assessment, 17/127 (13\%) patients were diagnosed with sarcopenia which comprised $6 / 17$ (35\%) severe sarcopenia $(4.7 \%$ of the total cohort). At the 6 months assessment, only $4 / 15$ (27\%) of the initial sarcopenic patients remained sarcopenic (3\% of the total cohort) and 3 of these 4 patients had severe sarcopenia. BMI (26,3 vs. $29,3, p=0.03$ ), COPD ( $20 \%$ vs. $3 \%, p=0.03$ ), comorbidities ( $93 \%$ vs. $67 \%, p=0.04$ ), total hospital stay duration (33 vs. 20 days $p=0.03$ ) and ICU stay duration (33 vs. 13 days, $p=0.01$ ) were significantly associated with sarcopenic vs. no sarcopenic patients. However, there was no significant difference concerning cardio-pulmonary evaluations between these two groups.

Conclusion: We here highlighted a sarcopenia prevalence at 3 and 6 months following a hospitalization for COVID-19 of $13 \%$ and $3 \%$ respectively, occurring mainly in patients with comorbidities. Sarcopenia was not associated to worse cardio-pulmonary results in comparison with non-sarcopenic patients.

\section{REFERENCES:}

[1] Di Filippo L, De Lorenzo R, D’Amico M, Sofia V, Roveri L, Mele R, et al. COVID-19 is associated with clinically significant weight loss and risk of malnutrition, independent of hospitalisation: A post-hoc analysis of a prospective cohort study. Clinical Nutrition [Internet]. oct 2020. https://linkinghub.elsevier. com/retrieve/pii/S0261561420305896

Disclosure of Interests: None declared

DOI: 10.1136/annrheumdis-2021-eular.2531

\section{AB0681 HOW COMMON IS COVID-19 IN CHILDREN, YOUNG PEOPLE AND ADULTS WITH RHEUMATIC DISEASES? RESULTS FROM THE INTERNATIONAL COVID-19 EUROPEAN PATIENT REGISTRY}

S. Shoop-Worrall ${ }^{1,2}$, S. Verstappen ${ }^{2,3}$, W. Costello ${ }^{4,5}$, S. Angevare ${ }^{4,6}$, Y. Uziel ${ }^{7,8}$ C. Wouters ${ }^{9,10}$, N. Wulffraat ${ }^{11}$, R. Beesley ${ }^{4,12}$. ${ }^{1}$ The University of Manchester, Centre for Health Infomatics, Manchester, United Kingdom; ${ }^{2}$ The University of Manchester, Centre for Epidemiology Versus Arthritis, Manchester, United Kingdom; ${ }^{3}$ Manchester University NHS FT, Manchester Academic Health Science Centre, NIHR Manchester BRC, Manchester, United Kingdom; ${ }^{4}$ ENCA, European Network for Children with Arthritis, Geneva, Switzerland; ${ }^{5}$ iCAN Ireland, iCAN Ireland, Bansha, Ireland; ${ }^{6} \mathrm{KAISZ}, \mathrm{KAISZ}$, Amsterdam, Netherlands; ${ }^{7}$ Meir Medical Center, Pediatric Rheumatology Unit, Kefar Sava, Israel; ${ }^{8}$ Sackler School of Medicine, Department of Pediatrics, TelAviv, Israel; ${ }^{9}$ University Hospitals Leuven, Pediatric Rheumatology Division, Leuven, Belgium; ${ }^{10} \mathrm{KU}$ Leuven University, Department of Microbiology and Immunology, Leuven, Belgium; ${ }^{11}$ Wilhelmina Children's Hospital (WKZ), Department of Pediatric Rheumatology, Utrecht, Netherlands; ${ }^{12}$ Juvenile Arthritis Research, Juvenile Arthritis Research, Tonbridge, United Kingdom

Background: People with rheumatic diseases may be at increased risk of contracting COVID-19 due to their rheumatic disease or immunosuppressive treatments. It is currently unclear what the COVID-19 disease burden is for these people and whether any of their personal or disease characteristics are associated with contracting COVID-19.

Objectives: To explore the proportion of, and characteristics associated with, contracting COVID-19 in children and young people (CYP) with rheumatic diseases and adults with rheumatic diseases from March 2020 to December 2020 during the COVID-19 pandemic.

Methods: CYP and adults recruited to the international COVID-19 European Patient Registry, a parent-led, online, self-referred prospective cohort recruiting participants from around the globe, were included in current study if enrolled between $20^{\text {th }}$ March 2020 and $30^{\text {th }}$ December 2020. Demographic information was collected at enrolment and rheumatic disease, diagnoses of COVID-19 and lifestyle factors were collected at weekly intervals.

The proportion of CYP and adults diagnosed with COVID-19 were assessed separately. Associations between contraction of COVID-19 at any point over follow-up and participant demographics, rheumatic disease and lifestyle factors at enrolment were assessed descriptively and via Mann-Whitney U-tests, Chisquared tests and Fisher's exact tests.

Results: Within 642 CYP and 3646 adults, the majority were female (67\%, 89\%) and most commonly from the UK $(43 \%, 82 \%)$, respectively. The most frequent diagnoses were polyarticular JIA (37\%) in the CYP cohort and RA in the adults (63\%). Comorbidities were common $(45 \%, 61 \%)$ and the majority were taking one or more immunosuppressive therapies (88\%, $92 \%)$, respectively. At the time of enrolment, $51 \%$ and $54 \%$ were practising social distancing, respectively. In both cohorts $\sim 3 \%$ contracted COVID-19 at some point during follow-up ( $n=18$ $(2.8 \%)$ in CYP and $n=103(2.8 \%)$ in the adult cohort).

In CYP, those who contracted COVID-19 were older (no COVID, median: 10, IQR: 7, 13, vs COVID, median: 14, IQR: 12, 16, p<0.001) and less often had oligoarticular JIA (no COVID: $31 \%$, COVID: $22 \%$ ) or polyarticular JIA (No COVID: $38 \%$, COVID: $11 \%$ ). Systemic JIA (no COVID: $7 \%$, COVID: 11\%) and enthesitis-related JIA (no COVID: $5 \%$, COVID: $22 \%$ ) were more common in those who contracted COVID. No other differences between those with and without COVID-19 were observed with respect to country of residence $(p=0.335)$, gender $(p=0.624)$, control of rheumatic disease $(p=0.459)$, comorbidities $(p=0.752)$, immunosuppressive medication $(p=0.713)$ or social distancing $(p=0.729)$. 